\title{
Perfect Pipelining for Streaming Large File in Peer-to-Peer Networks
}

\author{
Fei Chen and Xiaowei Wu \\ The University of Hong Kong
}

\begin{abstract}
We study the efficiency of large file streaming in a peer-topeer network in which a large file is cut into many pieces of equal size, and initially all pieces are known only by one source node. We analyze the number of rounds required, called the finishing time, for all nodes in the network to collect all pieces in the default order.

Based on the basic PUSH-PULL protocol, we design the Constant Out-degree Protocol (COP). At the beginning of the protocol, each node selects a constant number of neighbors, with only whom communication will be initiated. We focus our analysis on the performance of COP on preferential attachment graphs, which are believed to model peer-to-peer networks well. We show that a tight bound of $\Theta(B+\log n)$ rounds can be achieved with high probability for streaming $B$ pieces in preferential attachment graphs with $n$ nodes. Moreover, we show that there is a dichotomy in the results depending on how neighbors are contacted in each round; specifically, when each node avoids repeating initiation with neighbors in the previous $M \geq 2$ rounds, then the finishing time is improved to $\Theta\left(B+\frac{\log n}{\log \log n}\right)$ with high probability.

For lower bounds, we show that there is a class of regular graphs in which perfect pipelining is impossible for any PUSH-PULL protocols using random neighbor selection.
\end{abstract}

\section{Introduction}

The problem of information disseminating in a distributed network has been extensively studied. It is assumed that this information, usually called a rumor, was known by only one node in a large connected network. The goal is to disseminate this rumor to all nodes in the network, with bounded bandwidth and number of communications. Many protocols have been proposed based on various network topologies [71415]. Among these protocols, distributed algorithms have gained more attention since each node does not require global information about the network.

A gossip based protocol was first introduced by Demers et al. [7] for maintaining replicated databases. In this protocol, each node is only allowed to communicate with its own neighbors in each round. A similar distributed protocol called PUSH-PULL protocol was introduced later. In this protocol, each node initiates communication with one of its neighbors per round; the node pushes the rumor to the neighbor if it is informed of the rumor, or pulls the rumor from

J. Diaz et al.(Eds.): TCS 2014, LNCS 8705, pp. 27-38 2014.

(C) IFIP International Federation for Information Processing 2014 
the neighbor if the neighbor is informed. A common performance measure of protocols is the number of rounds required to have all nodes informed, which is called the finishing time.

We consider the rumor spreading problem when the rumor is not a tiny piece of information, but a large file which can not be transmitted from one node to another in one round. In this case, we divide the file into $B$ small pieces so that each piece can be transmitted in one round. This problem can also be considered as the multiple-rumor version of the rumor spreading problem with streaming requirement. We analyse the finishing time for all nodes in the network to collect all $B$ pieces in the correct order. For instance, in peer-to-peer video streaming, a node needs to receive earlier portions first and the next portion needs to arrive before all currently received portions have been played. We consider two properties of a protocol that are important for streaming multiple pieces: perfect pipelining and ordered pieces transmission, which we explain below.

\subsection{Our Contribution and Results}

We study the large file streaming problem in which $B$ ordered pieces of information known to one source node are to be spread in a graph with $n$ nodes. We consider protocols that have the following properties.

1. Loosely speaking, a (randomized) protocol for multiple pieces spreading achieves perfect pipelining on $G(V, E)$ if the following holds: if the finishing time for spreading one piece is $T$ (with high probability), then the finishing time for spreading $B$ pieces is $O(B+T)$ (with high probability) in $G$.

2. A protocol employs ordered pieces transmission if the pieces are transmitted and received in the correct order.

Our results and contribution are summarized as follows.

Achieving perfect pipelining with COP. In Section 4, we modify the basic PUSH-PULL protocol and design the Constant Out-degree Protocol (COP). At the beginning of the protocol, each node selects a constant number of neighbors, with only whom communications will be initiated.

We show that using COP, steaming $B$ pieces in preferential attachment graphs requires $O(B+\log n)$ rounds, which achieves perfect pipelining. We also prove a tight lower bound $\Omega(B+\log n)$ for the finishing time. As considered by Doerr et al. [8], if each node does not repeat neighbor initiation from the previous 2 rounds, COP can achieve finishing time $O\left(B+\frac{\log n}{\log \log n}\right)$, which is also tight. This theoretical gap in the finishing time between the two cases suggests that after the random neighbor selection phase to identify out-going neighbors, deterministic round-robin for the pieces streaming phase is optimal.

We briefly outline our technical contributions. According to Arthur and Panigrahy [1], spreading $B$ pieces in a graph with diameter $d$ and maximum degree $D$ using random PUSH requires $O(D(B+\max \{d, \log n\}))$ rounds with high probability (w.h.p.), no matter how the pieces are chosen for transmission. For preferential attachment graphs, the resulting upper bound $O(\sqrt{n}(B+\log n))$ is loose 
because the maximum degree is as large as $\Omega(\sqrt{n})$. However, the large degree nodes are crucial for single piece spreading [8] since they are highly connected. To overcome this technical hurdle, our protocol (COP) is based on the following two observations: (1) for single piece spreading, even if each node is "active" for only a constant number of rounds, w.h.p., the piece can still reach all nodes via paths of length $O\left(\frac{\log n}{\log \log n}\right) ;(2)$ we can direct the edges to achieve constant out-degree, even when the underlying undirected graph has a high maximum degree; this allows us to achieve the same result as in [1] if we use both PUSH and PULL.

Implication on the Performance of Streaming. We show that our protocol achieves tight upper bound $\Theta(B+\log n)$ when the ordered pieces transmission is adopted. In this case, all nodes will receive all pieces of the file in the correct order and perfect pipelining is achieved. As far as we know, this is the first time perfect pipelining is proved for streaming large file in peer-to-peer network.

Impossibility of Perfect Pipelining in Some Cases. In Section 3 we consider the basic PUSH-PULL protocol for streaming $B$ pieces in complete graphs. Unfortunately, perfect pipelining cannot be achieved with this simple protocol. In Section 5, we give a lower bound for the finishing time in a particular class of D-regular graphs, called Necklace Graphs. We show that spreading $B$ pieces under any PUSH-PULL protocol in which communication partners are chosen uniformly at random in each round (no matter how those pieces are selected for transmission) in Necklace Graph requires $\Omega(D(B+d))$ rounds, where $d$ is the diameter, for any $\omega(1) \leq D \leq o(n)$.

\subsection{Related Work}

The single piece (usually called a rumor) spreading problem has been extensively studied. Using the PUSH-PULL protocol, the finishing time (also called stopping time) is $\Theta(\log n)$ for both complete graphs [16 13] and random graphs [910]. Rumor spreading in preferential attachment graphs (PA-graphs) was also studied and two tight upper bounds $O(\log n)$ and $O\left(\frac{\log n}{\log \log n}\right)$ for different protocols were shown by Doerr et al. [8. Given a graph with conductance $\phi$, an $O\left(\phi^{-1} \log n\right)$ upper bound was proved by Giakkoupis [11, which is tight. For rumor spreading in general graphs, Censor-Hillel et al. 4] gave an algorithm which constructs subgraphs along with the rumor spreading process and showed that the finishing time is at most $O(d+$ poly $\log n)$, where $d$ is the diameter of the underlying graph, with no dependence on the conductance.

Although observed by experiments [5] and extensively applied on the Internet, the high efficiency of multiple-rumor spreading is hardly analyzed. Deb et al. [6] presented a protocol based on random linear network coding and showed that in the complete graph spreading $B=\Theta(n)$ rumors requires at most $O(n)$ rounds, which is asymptotically optimal. Also using network coding, Haeupler [12] gave a protocol that achieves perfect pipelining in many other graphs. In their protocol, communication partners exchange a random linear combination of rumors and they show that after receiving enough combinations, all rumors can be decoded locally. 
Given a graph with diameter $d=\Omega(\log n)$ and maximum out-degree $D$, an $O(D(B+d))$ upper bound on the finishing time was proved by Arthur and Panigrahy [1] for spreading $B$ rumors with PUSH. Since $\Omega(B+d)$ is a trivial lower bound, this upper bound is tight when $D$ is a constant. This result actually inspired the construction of our protocol COP, which attempts to find a subgraph that has constant out-degree and small diameter in the first step.

\section{Preliminaries}

Consider a graph $G$ with $n$ nodes and $B$ pieces indexed by $\{1,2, \ldots, B\}$; for streaming application, we assume that smaller index means higher priority. Throughout this paper we suppose that initially a single source node $u_{0} \in V$ has all $B$ pieces while other nodes have no pieces. We say a node is waiting if it has no pieces, downloading if it has at least one piece, and finished if it has all the pieces. We use log to denote the logarithm with base 2 .

Pieces are transmitted in a round-by-round manner. During a round of the pieces streaming process, we say there is a piece transmission between two nodes if some piece is transmitted from one node to the other. The finishing time is the number of rounds required until all nodes in the graph become finished.

The Basic PUSH-PULL Protocol. For each node $u \in V$ and integer $t \geq 1$, let $p_{t}^{u}$ be the current downloading position of $u$ at time $t$. Note that $p_{0}^{u}=B$ if $u=u_{0}$, and $p_{0}^{u}=0$ otherwise. In each round each node independently selects a neighbor uniformly at random, and initiates communication with that neighbor. In round $t \geq 1$, for an instance of communication initiated by $u$ between $u$ and $v$, the transmission follows the following rules.

1. If $p_{t-1}^{u}=p_{t-1}^{v}$, then no transmission is made;

2. If $p_{t-1}^{v}>p_{t-1}^{u}$, then node $u$ pulls from node $v$ the piece with index $p_{t-1}^{u}+1$;

3. If $p_{t-1}^{v}<p_{t-1}^{u}$, then node $u$ pushes to node $v$ the piece with index $p_{t-1}^{v}+1$.

Note that each node initiates communication with exactly one neighbor in each round and at most one piece is transmitted. Also note that all $B$ pieces are initiated by the source node at the beginning and hence each node in the graph will receive all the pieces in the fixed order $1,2, \ldots, B$.

In Section 3, we show that the basic PUSH-PULL protocol cannot achieve perfect pipelining on the complete graph; in Section 4, we modify the basic PUSH-PULL protocol and design the Constant Out-degree Protocol, which achieves perfect pipelining for directed random graphs and preferential attachment graphs.

\section{Low Efficiency of Basic PUSH-PULL}

To motivate why we consider COP, we analyze the performance of the basic PUSH-PULL protocol on complete graph $K_{n}$ with source node $u_{0}$ having all $B$ pieces. We show that perfect pipelining can not be achieved since (on average) each node has to wait for $\Omega(\log n)$ rounds to get the next piece. 
Theorem 1. The finishing time for streaming $B$ pieces from one source node in a complete graph with $n$ nodes using the basic PUSH-PULL protocol is $\Omega(B \log n)$ with probability $1-\exp (-\Theta(B))$.

Note that by experiments, Theorem 1 is not true if the pieces are spread randomly. We conjecture that the finishing time for spreading $B$ pieces with random pieces selection is $O(B+\log n)$. The proof of Theorem 1 is included in the full version. Intuitively, when all nodes in the graph are waiting for the same piece, the efficiency is low since most of the communications are useless. However, if we can guarantee that at least a constant fraction of communications are useful, then after $O(B)$ rounds, all nodes will become finished. Following this idea, we define in Section 4 a protocol that constructs a constant maximum outdegree subgraph before applying the basic PUSH-PULL protocol. We show that in several cases, the subgraph constructed by our protocol has small diameter and using basic PUSH-PULL protocol, perfect pipelining can be achieved.

\section{Constant Out-Degree Protocol}

Constant Out-degree Protocol. Let $D$ be a fixed constant parameter used in the protocol. Suppose we are given an input graph with one source node.

(1) Out-going Edge Selection Phase. A directed subgraph $H$ is formed, where each node $u$ chooses independently its outgoing edges and outgoing neighbors, with only whom node $u$ will initiate communications in the next phase. We consider all input graphs as multi-graphs. Each node $u$ chooses $\min \{D, \operatorname{deg}(u)\}$ (out-going) edges incident to $u$ uniformly at random without replacement. The chosen edges are the outgoing edges and the incident nodes (excluding $u$ ) are the outgoing neighbors of $u$ in $H$.

(2) Pieces Streaming Phase. In each round, each node chooses one of its sampled outgoing edges uniformly at random to initiate communication. In round $t \geq 1$, for an instance of communication initiated by $u$ between nodes $u$ and $v$, the transmission follows as the basic PUSH-PULL protocol.

In other words, we construct a directed subgraph $H$ and apply the basic PUSH-PULL protocol to $H$, where the directions of initiation coincide with the directions of edges. Since the only difference between COP and the basic PUSHPULL protocol is an extra neighbor selection process, we know that if perfect pipelining can be achieved in the (random) subgraph $H$ using basic PUSH-PULL, then perfect pipelining is achieved by COP in the input graph. We show in this section that perfect pipelining can be achieved by applying COP to stream multiple pieces in directed random graphs and preferential attachment graphs. Unless otherwise specified, the diameter of a graph refers to that of the underlying induced undirected graph.

Deterministic Rumor Spreading Phase. Suppose we consider the case that after the random subgraph $H$ is constructed in the first phase each node initiates communication with its outgoing neighbors in a round-robin way. Recall that since the out-degree of the subgraph $H$ is at most $D$, there is communication 
between two neighboring nodes at most every $D$ rounds. It follows that if the diameter of $H$ is at most $d$, all nodes will become finished after $D(B+d)$ rounds.

Fact 1. Given any input graph with $n$ nodes, if the underlying undirected graph of the subgraph constructed by COP with constant parameter D has diameter $d$ and each node contacts its neighbors in a round-robin way, then for all $B \geq 1$, the finishing time for streaming $B$ pieces starting from one source node $u_{0}$ in the input graph using COP with parameter $D$ is at most $D(B+d)=O(B+d)$.

Note that Fact 1 holds with probability 1 since it is deterministic. If the diameter of the graph is $\Omega(\log n)$, the random neighbor chosen process achieves a similar upper bound w.h.p.. We use the following fact [1, Proposition 4.1] on streaming multiple pieces along a path.

Fact 2 (Streaming Multiple Pieces on a Path). Suppose there exists a path $P\left(v_{0}, v_{s}\right)=\left\{v_{0}, v_{1}, \ldots, v_{s}\right\}$ of length s between two nodes $v_{0}$ and $v_{s}$ such that in each round, communication between any two adjacent nodes $v_{i}$ and $v_{i+1}$ is performed with probability at least $p$ independently for each pair of adjacent nodes. Assume only $v_{0}$ has all $B$ pieces and all other nodes are waiting. Then if $s^{\prime} \geq s$, with probability $1-2 \exp \left(-\frac{s^{\prime}}{2}\right)$, node $v_{s}$ will become finished after $\frac{4\left(B+4 s^{\prime}\right)}{p}$ rounds.

The fact above implies that by using COP protocol, perfect pipelining can be achieved in any graph if the subgraph $H$ constructed has a small diameter $d$.

Theorem 2. Suppose the subgraph constructed by COP has diameter d, then for all $B \geq 1$, the finishing time for streaming $B$ pieces starting from one source node $u_{0}$ is at most $O(B+\max \{d, \log n\})$ with probability $1-O\left(\frac{1}{n}\right)$.

Proof. We denote the subgraph constructed by COP with constant parameter $D$ by $H$, which has diameter $d$. Hence for any node $v$, there exists a path $P=$ $\left(u_{0}, u_{1}, \ldots, u_{s-1}, u_{s}=v\right)$ in the undirected graph induced by $H$ between $u_{0}$ and $v$, where $s \leq d$ is the length of the path.

Hence, in the directed graph $H$, for any $i=\{0,1, \ldots, s-1\}$, there is a directed edge between nodes $u_{i}$ and $u_{i+1}$. Since both nodes $u_{i}$ and $u_{i+1}$ have out-degree at most $D$, which is a constant, communication between $u_{i}$ and $u_{i+1}$ will be performed with probability at least $\frac{1}{D}$ in each round. However, if there exists two directed edges $\left(u_{i}, u_{i+1}\right)$ and $\left(u_{i}, u_{i-1}\right)$ that start at $u_{i}$, then the communications between $u_{i}$ and $u_{i+1}, u_{i}$ and $u_{i-1}$ are not independent (only one of them can happen in each round). To make the communications independent, we define phases such that each phase consist of two rounds. In each phase, for each $i=\{1, \ldots, s-1\}$, we assume that the communication between $u_{i}$ and $u_{i+1}$ is invalid in the first round and the communication between $u_{i}$ and $u_{i-1}$ is invalid in the second round. Note that the assumption will only make the downloading process slower. Under this assumption, we know that for each $i=\{0,1, \ldots, s-1\}$, in each phase, communication between $u_{i}$ and $u_{i+1}$ will be performed with probability at least $\frac{1}{D}$, independently.

Hence, for each node $v$ in $H$, there is a path of length $s \leq d$ between $u_{0}$ and $v$ such that in each phase, communications between any two adjacent nodes 
will be performed with probability at least $\frac{1}{D}$, independently for each pair of adjacent nodes. Hence by Fact 2 , if $s^{\prime} \geq s$, with probability $1-2 \exp \left(-\frac{s^{\prime}}{2}\right)$, node $v$ will become finished after $4 D\left(B+4 s^{\prime}\right)$ phases. If $s \geq 4 \log n$, then set $s^{\prime}=s$ and node $v$ will become finished after $4 D(B+4 s)=O(B+d)$ phases with probability $1-O\left(\frac{1}{n^{2}}\right)$. If $s<4 \log n$, then set $s^{\prime}=4 \log n$ and node $v$ will become finished after $4 D(B+16 \log n)=O(B+\log n)$ phases with probability $1-O\left(\frac{1}{n^{2}}\right)$. Therefore, with probability $1-O\left(\frac{1}{n^{2}}\right)$, node $v$ will become finished after $O(B+\max \{d, \log n\})$ phases, which means $O(B+\max \{d, \log n\})$ rounds. A union bound on $n-1$ nodes in $H$ yields the result.

By Theorem 2, we know that the finishing time of COP is highly related to the diameter $d$ of the subgraph constructed in the first step. If $d=\Omega(\log n)$, then using round-robin in the pieces streaming phase has the same bound as random neighbor selection. However, as we shall later see, the PA-graphs have diameter $O\left(\frac{\log n}{\log \log n}\right)$ w.h.p., and indeed we show that there is a dichotomy in the finishing times between deterministic and random pieces streaming phases.

COP on Directed Random Graphs. We apply COP with $D=8$ to stream $B$ pieces in directed random graphs $G_{n, p}$ with $p \geq \frac{6 \log n}{n}$. Observe that the neighbor selection phase actually constructs a random $D$-out-degree subgraph. For space reasons, we show in our full version that a random $D$-out-degree graph induces an expander with diameter $O(\log n)$. Hence the following theorem follows immediately; its proof is also presented in the full version.

Theorem 3 (Finishing Time of COP in $G_{n, p}$ ). For all $B \geq 1$, the finishing time for streaming $B$ pieces starting from one source node in directed random graphs $G_{n, p}$ with $n$ nodes and $p \geq \frac{6 \log n}{n}$ using the constant out-degree protocol with $D=8$ is at most $O(B+\log n)$ with probability $1-O\left(\frac{1}{n}\right)$.

The intuition behind the proof can be summarized as follows.

1. When $p \geq \frac{6 \log n}{n}$, the random subgraph construction can be equivalently modelled as in complete graph.

2. For any node $u$, the number of nodes reachable from $u$ grows exponentially as the distance increases.

3. For any two nodes $u$ and $v$, if both of them have many reachable nodes within a distance of $O(\log n)$, then it is very likely that there exists a common node $w$ that is reachable from both $u$ and $v$ by paths with length $O(\log n)$.

Next we consider the finishing time of multiple-piece streaming in Preferential Attachment graphs (PA-graphs). The notion of preferential attachment graphs was first introduced by Barabási and Albert [2], and they have been used to model social and peer-to-peer networks. In this work, we follow the formal definition by Bollobás et al. [3]. Let $G_{m}^{n}$ be a PA-graph. We denote by $\operatorname{deg}_{G}(v)$ the degree of a vertex $v$ in $G_{m}^{n}$.

Definition 1 (Preferential Attachment Graphs). Let $m \geq 2$ be a fixed constant parameter. The random graph $G_{m}^{n}$ is an undirected graph on the vertex set $V:=\{1, \ldots, n\}$ inductively defined as follows. 
1. $G_{m}^{1}$ consists of a single vertex with $m$ self-loops.

2. For all $n>1, G_{m}^{n}$ is built from $G_{m}^{n-1}$ by adding the new node $n$ together with $m$ edges $e_{n}^{1}=\left\{n, v_{1}\right\}, \ldots, e_{n}^{m}=\left\{n, v_{m}\right\}$, inserted one after the other in this order. Let $G_{m, i-1}^{n}$ denote the graph right before the edge $e_{n}^{i}$ is added. Let $M_{i}=\sum_{v \in V} \operatorname{deg}_{G_{m, i-1}^{n}}(v)$ be the sum of the degrees of all the nodes in $G_{m, i-1}^{n}$. The endpoint $v_{i}$ is selected randomly such that $v_{i}=u$ with probability $\frac{\operatorname{deg}_{G_{m, i-1}^{n}}^{n}(u)}{M_{i}+1}$, except for $n$ that is selected with probability $\frac{\operatorname{deg}_{G_{m, i-1}^{n}}^{n}(n)+1}{M_{i}+1}$.

It can be easily seen that for $m=1$ the graph is disconnected w.h.p.; so we focus on the case $m \geq 3$. For each node $i$ and $k \in[m]$, let $\tau_{i, k}$ be the node chosen by $i$ in the $k$-th rounds after $i$ is added to $G_{m}^{n}$. We call the first node added to $G_{m}^{n}$ node $v_{1}$. Bollobás et al. [3] introduced an equivalent way of generating $G_{m}^{n}$ that is more accessible. In that model, each node $i$ is assigned a random variable $\omega_{i} \in(0,1)$ following some distribution and edges are added to node $i$ with probability proportional to $\omega_{i}$. The formal definition of the new model is given in [3]. Under this equivalent model, a node $i$ is called useful if $\omega_{i} \geq \frac{\log ^{2} n}{n}$ and non-useful otherwise.

We apply COP with $D=3$ to stream $B$ pieces in PA-graph and show that the finishing time in a PA-graph is at most $O(B+\log n)$. Note that the PA-graph is a multi-graph and the outgoing neighbors of a node is a multiset in the subgraph constructed by COP.

Theorem 4 (Finishing Time of COP In PA-graphs). For all $B \geq 1$, the finishing time for streaming $B$ pieces starting from any source node in a PA-graph $G_{m}^{n}$ using the constant out-degree protocol with $D=3$ is at most $O(B+\log n)$ with probability $1-o(1)$.

Communication Initiation without Repeat. In each round of the downloading process, each node initiates communication uniformly at random with an outgoing neighbor that is not initiated with in the last $\min \left\{M, \operatorname{deg}_{G}(u)-1\right\}$ rounds; for a multi-set of outgoing neighbors, multiplicities are respected. For $D=3$ and $M=2$, this is equivalent to round-robin neighbor selection.

Lemma 1. [8, Lemma 5.8] There exists a property $\mathcal{P}$ of $G_{m}^{n}$ that holds with probability $1-o(1)$ such that conditioning on $\mathcal{P}$, with probability at least 1 $n^{-\Omega(n)}$, using only PUSH on $G_{m}^{n}$ (avoiding repeated neighbor initiation from the previous $M \geq 2$ rounds), a piece initiated at a node $u$ reaches a useful node in $O(\log \log n)$ rounds.

Lemma 2. [8, Lemma 5.11] If each node $v$ in $G_{m}^{n}$ receives an independent random variable $x_{v}$ such that $x_{v}=1$ with constant probability $p$ and $x_{v}=0$ otherwise, then with probability $1-o(1)$, for each useful node $u$, there exists a path of length $O\left(\frac{\log n}{\log \log n}\right)$ between $u$ and node $v_{1}$ such that every second node $v$ in the path has degree $m$, its two neighbors in the path are $\tau_{v, 1}$ and $\tau_{v, 2}$ and has $x_{v}=1$.

Note that all $x_{v}$ 's are independent and the randomness of $x_{v}$ comes from the behavior of node $v$. In the rest of the section, we assume the PA-graph $G_{m}^{n}$ under consideration has the properties stated above. 


\subsection{Upper Bound for the Stopping Time}

First, it is obvious that each node in $G_{m}^{n}$ has $\operatorname{deg}_{G}(u) \geq m \geq 3$ and the outdegree of each node in $H$ is $\min \left\{D, \operatorname{deg}_{G}(u)\right\}=3$, for the case $D=3$. A node $v$ in $H$ is called a fast node if $\operatorname{deg}_{G}(v)=m$ and it chooses both $\tau_{v, 1}$ and $\tau_{v, 2}$ as its outgoing neighbors in $H$.

In Lemma 3. we show that for each non-useful node $v$, there is at least one useful node $u$ that can be reached from $v$ by a directed path of length $O(\log \log n)$. In Lemma 4, we show that all useful nodes are connected to node $v_{1}$ by paths of length $O\left(\frac{\log n}{\log \log n}\right)$ in $H$ and every second node in the paths is fast. We include the proof of Lemma 4 in our full version. These two lemmas imply that the diameter of $H$ is at most $O\left(\frac{\log n}{\log \log n}\right)$. Given a node $u$ and an integer $k$, we denote by $H_{k}^{u}$ the set of nodes that can be reached by a directed path of length at most $k$ in the subgraph $H$.

Lemma 3 (Useful Node in $O(\log \log n)$-Neighborhood). With probability $1-o(1)$, for each non-useful node $u$, at least one useful node will be included in $H_{K}^{u}$ for some $K=O(\log \log n)$ following the constant out-degree protocol.

Proof. We shall use Lemma 1 [8, Lemma 5.8] to prove this result. We condition on the same property $\mathcal{P}$ on $G_{m}^{n}$ as in Lemma 19. In the proof of [8, Lemma 5.8], the authors consider a restricted version of PUSH strategy on $G_{m}^{n}$ with one piece starting at a fixed node $u \in V$. We show that there is a correspondence between their restricted piece streaming process and our outgoing neighbor selection process. Note that in the proof of [8, Lemma 5.8], the authors assume that each node performs only PUSH and each node is active for only 3 rounds after it start downloading. With the memory property, this means the node contacts three random distinct neighbors in those 3 rounds (if the node has degree at least 3 ); this corresponds to the selection of $D=3$ outgoing neighbors uniformly at random in COP.

Hence, the single rumor spreading process in [8, Lemma 5.8] can be coupled with our outgoing neighbor selection phase such that if a node $v$ becomes downloading after $3 K$ rounds in [8, Lemma 5.8], then there is a path from $u$ to $v$ of length $K$ such that the next node in the path is an outgoing neighbor of the previous node selected by COP. With this coupling argument, we know that conditioning on property $\mathcal{P}$, with probability $1-n^{-\Omega(n)}$, a useful node will be included in $H_{K}^{u}$ for some $K=O(\log \log n)$. By union bound on all nodes in $V$, the conditional probability that this holds for all $H_{K}^{u}$ 's is at least $1-n^{-\Omega(n)} \cdot n$. Since property $\mathcal{P}$ holds with probability at least $1-o(1)$, our result follows.

Lemma 4 (Distances Between Useful Nodes). With probability $1-o(1)$, for each useful node $u$, there exists a path of length $O\left(\frac{\log n}{\log \log n}\right)$ between $u$ and 1 such that every second node in the path is fast.

Proof of Theorem 4; By Lemma 3 and 4 we know that with probability $1-o(1)$, each non-useful node can reach a useful node by a path of length at most $O(\log \log n)$ and all useful nodes are connected to node $v_{1}$ by paths of length 
at most $O\left(\frac{\log n}{\log \log n}\right)$ in $H$, which implies that the underlying undirected graph of $H$ has diameter at most $O(\log \log n)+O\left(\frac{\log n}{\log \log n}\right)=O\left(\frac{\log n}{\log \log n}\right)$. By Theorem 2 the finishing time of streaming $B$ pieces in a PA-graph $G_{m}^{n}$ using COP with $D=3$ is at most $O(B+\log n)$ with probability $1-o(1)-O\left(\frac{1}{n}\right)=1-o(1)$.

\subsection{Lower Bound for the Stopping Time}

We have shown that streaming $B$ pieces in a PA-graph $G_{m}^{n}$ using COP with $D=3$ requires at most $O(B+\log n)$ rounds. However, the PA-graph and the subgraph $H$ of the PA-graph have diameter $O\left(\frac{\log n}{\log \log n}\right)$, which is $o(\log n)$. Note that by Fact 2, each node $v$ will become finished after $O\left(B+\frac{\log n}{\log \log n}\right)$ with probability $1-\exp \left(-\frac{\log n}{\log \log n}\right)$. However, since the failure probability is $\omega\left(\frac{1}{n}\right)$ and there are $n$ nodes, we cannot use union bound to show that w.h.p., all nodes will be finished after $O\left(B+\frac{\log n}{\log \log n}\right)$ rounds. Indeed, we show that the finishing time of streaming $B$ pieces in a PA-graph $G_{m}^{n}$ using COP with $D=3$ needs $\Omega(B+\log n)$ rounds, which implies that the upper bound we have proved in the last section is tight and perfect pipelining is achieved. More specifically, we prove the following result.

Theorem 5 (Lower Bound For COP in PA-Graphs). With probability $1-o(1)$, COP with any constant parameter $D$ needs $\Omega(B+\log n)$ rounds to get all nodes in a PA-graph $G_{m}^{n}$ finished.

Proof. It is obvious that if the protocol needs $\Omega(\log n)$ rounds to spread 1 piece to all nodes in $G_{m}^{n}$, then $\Omega(B+\log n)$ rounds are required for the same protocol to stream $B$ pieces to all nodes in $G_{m}^{n}$. Hence, we only consider the case when $B=1$ in the following proof.

By the proof of [8, Theorem 3.3], we know that with probability $1-o(1)$, there are $\Omega(n)$ edges in $G_{m}^{n}$ whose incident nodes are of degree at most $m+c$, where $c$ is a constant. We call those edges links and let $L \subseteq E$ be the collection of links, where $|L|=\Omega(n)$. Note that for any two edges $e_{1}$ and $e_{2}$ in $L, e_{1}$ and $e_{2}$ may have common endpoints or the endpoints of $e_{1}$ and $e_{2}$ may have common neighbors. To avoid this and make the proof simpler, we remove one of those two edges if such a situation happens. Let $\tilde{L}$ be the collection of links after the removals. Since each endpoint of a link is of degree at most $m+c$, we conclude that $|\tilde{L}| \geq \frac{1}{(m+c-1)^{2}+1}|L|=\Omega(n)$ (to keep one edge, at most $(m+c-1)^{2}$ edges will be deleted). Note that any two links in $\tilde{L}$ have no common endpoint and the endpoints of any two edges have no common neighbor.

Fix one link in $\tilde{L}$, denoted by $(u, v), u$ will choose $v$ as an outgoing neighbor with probability at least $\frac{D}{m+c}$ and $v$ will choose $u$ as an outgoing neighbor also with probability at least $\frac{D}{m+c}$, independently in COP. Hence for each of those links, with constant probability, the two endpoints of the link will choose each other as outgoing neighbor in the protocol, independently for all links in $\tilde{L}$. If that happens, we call the link preserved in $H$. By Chernoff bound, we know that with probability $1-\exp (-\Omega(n))$, there are $\Omega(n)$ preserved links. 
Fix one pair of nodes $(u, v)$ that are connected by a preserved link, in each round, if the two nodes are waiting, then with probability at least $\left(\frac{1}{D}\right)^{2}(1-$ $\left.\frac{1}{D}\right)^{2(D-1)}=\delta$, they will remain waiting. Note that $\delta$ is a constant. The probability that $(u, v)$ remains waiting for $\frac{\log n}{2 \ln \delta^{-1}}$ is therefore at least $n^{-\frac{1}{2}}$. Since each of those pairs are disjoint and the choices of communications are made independently, the probability that none of the $\Omega(n)$ pairs remains waiting after $\frac{\log n}{2 \ln \delta^{-1}}$ is at most $\left(1-n^{-\frac{1}{2}}\right)^{\Omega(n)} \leq \exp \left(-n^{-\frac{1}{2}} \Omega(n)\right) \leq \exp \left(-\Omega\left(n^{\frac{1}{2}}\right)\right)$. Hence with probability $1-o(1)-\exp (-\Omega(n))-\exp \left(-\Omega\left(n^{\frac{1}{2}}\right)\right)=1-o(1)$, COP needs $\Omega(\log n)$ rounds to spread one piece to all nodes in $G_{m}^{n}$ and $\Omega(B+\log n)$ rounds to stream $B$ pieces to all nodes in $G_{m}^{n}$ using COP with any constant parameter $D$.

\subsection{Improving the Upper Bound}

It is established by Doerr et al. 8] that if each node in $G_{m}^{n}$ avoid repeated neighbor initiation from the previous $M \geq 2$ rounds, the upper bound of the finishing time of spreading one piece in $\bar{G}_{m}^{n}$ can be improved to $O\left(\frac{\log n}{\log \log n}\right)$, which is tight up to a constant factor. Note that with $M=2$ and $D=3$, the pieces streaming phase of COP is the same as round-robin and by Fact 1] the next theorem follows immediately.

Theorem 6. For all $B \geq 1$, the finishing time for streaming $B$ pieces starting from any source node in a PA-graph $G_{m}^{n}$ using COP with round-robin pieces streaming phase is at most $O\left(B+\frac{\log n}{\log \log n}\right)$ with probability $1-o(1)$.

\section{Lower Bound}

In Section 4 we show that perfect pipelining can be achieved using protocols based on the basic PUSH-PULL protocol when streaming multiple pieces in directed random graphs and preferential attachment graphs. In this section we give a class of $D$-regular graphs, where $\omega(1) \leq D \leq o(n)$, with diameter $d$ and show that perfect pipelining is impossible in those regular graphs for any protocols in which communication partners are chosen uniformly at random in each round. We show that streaming $B$ pieces from one source node in those regular graphs requires at least $\Omega(D(B+d))$ rounds, no matter how the pieces are chosen to be transmitted. We denote those protocols by PUSH-PULL based protocols. Note that by the upper bound proved by [1, our lower bound is actually tight.

The Necklace Graph. A $D$-regular necklace graph $N(V, E)$ with $n$ nodes consists of $\ell:=\frac{n}{D+1}$ units $U_{1}, U_{2}, \ldots, U_{\ell}$. Each unit $U_{i}$ for $i \in[\ell]$ contains $D+1$ nodes, numbered by $1,2, \ldots, D+1$. Within a unit $U_{i}$, there is an edge between each pair of nodes except the pair $\{1, D+1\}$. That is, the subgraph of $N(V, E)$ induced by nodes in $U_{i}$ is $K_{D+1} \backslash 1, D+1$. For each $i \in\{1,2, \ldots, \ell-1\}$, the two units $U_{i}$ and $U_{i+1}$ are connected via an edge between node $D+1$ of $U_{i}$ and node 1 of $U_{i+1}$. Units $U_{\ell}$ and $U_{1}$ are connected via an edge between node $D+1$ of $U_{\ell+1}$ and node 1 of $U_{1}$. 
Theorem 7 (Lower Bound for PUSH-PULL). Let $D$ be an integer such that $\omega(1) \leq D \leq o(n)$. Let $N(V, E)$ be a D-regular necklace graph with $|V|=n$ and diameter $d$. Then for all $B \geq 1$, the finishing time for streaming $B$ pieces starting from one source node in $N(V, E)$ using any PUSH-PULL based protocol is at least $\Omega(D(B+d))$ with probability at least $1-o(1)$.

For space reasons, we put its proof in the full version. Theorem 7 indicates that perfect pipelining is impossible when streaming multiple pieces in necklace graphs using any PUSH-PULL based protocol.

\section{References}

1. Arthur, D., Panigrahy, R.: Analyzing bittorrent and related peer-to-peer networks. In: SODA 2006, pp. 961-969. ACM, New York (2006)

2. Barabasi, A.L., Albert, R.: Emergence of scaling in random networks. Science 286, 509-512 (1999)

3. Bollobás, B., Riordan, O.: The diameter of a scale-free random graph. Combinatorica 24(1), 5-34 (2004)

4. Censor-Hillel, K., Haeupler, B., Kelner, J., Maymounkov, P.: Global computation in a poorly connected world: fast rumor spreading with no dependence on conductance. In: STOC 2012. ACM, New York (2012)

5. Chan, H.-L., Lam, T.-W., Wong, P.W.H.: Efficiency of data distribution in bitTorrent-like systems. In: Kao, M.-Y., Li, X.-Y. (eds.) AAIM 2007. LNCS, vol. 4508, pp. 378-388. Springer, Heidelberg (2007)

6. Deb, S., Médard, M., Choute, C.: Algebraic gossip: a network coding approach to optimal multiple rumor mongering. IEEE/ACM Trans. Netw. 14(SI), 2486-2507 (2006)

7. Demers, A., Greene, D., Hauser, C., Irish, W., Larson, J., Shenker, S., Sturgis, H., Swinehart, D., Terry, D.: Epidemic algorithms for replicated database maintenance. In: PODC 1987. ACM Press, New York (1987)

8. Doerr, B., Fouz, M., Friedrich, T.: Social networks spread rumors in sublogarithmic time. In: STOC 2011. ACM, New York (2011)

9. Feige, U., Peleg, D., Raghavan, P., Upfal, E.: Randomized broadcast in networks. In: Asano, T., Imai, H., Ibaraki, T., Nishizeki, T. (eds.) SIGAL 1990. LNCS, vol. 450, pp. 128-137. Springer, Heidelberg (1990)

10. Fountoulakis, N., Huber, A., Panagiotou, K.: Reliable broadcasting in random networks and the effect of density. In: INFOCOM 2010, pp. 2552-2560. IEEE Press, Piscataway (2010)

11. Giakkoupis, G.: Tight bounds for rumor spreading in graphs of a given conductance. In: STACS 2011, vol. 9, pp. 57-68. Schloss Dagstuhl-Leibniz-Zentrum fuer Informatik, Dagstuhl (2011)

12. Haeupler, B.: Analyzing network coding gossip made easy. In: STOC 2011, pp. 293-302. ACM, New York (2011)

13. Karp, R., Schindelhauer, C., Shenker, S., Vocking, B.: Randomized rumor spreading. In: FOCS 2000, p. 565. IEEE Computer Society, Washington, DC (2000)

14. Kempe, D., Kleinberg, J., Demers, A.: Spatial gossip and resource location protocols. In: STOC 2001, pp. 163-172. ACM, New York (2001)

15. Kempe, D., Kleinberg, J.M.: Protocols and impossibility results for gossip-based communication mechanisms. In: FOCS 2002, pp. 471-480. IEEE Computer Society, Washington, DC (2002)

16. Pittel, B.: On spreading a rumor. SIAM J. Appl. Math. 47(1) (March 1987) 